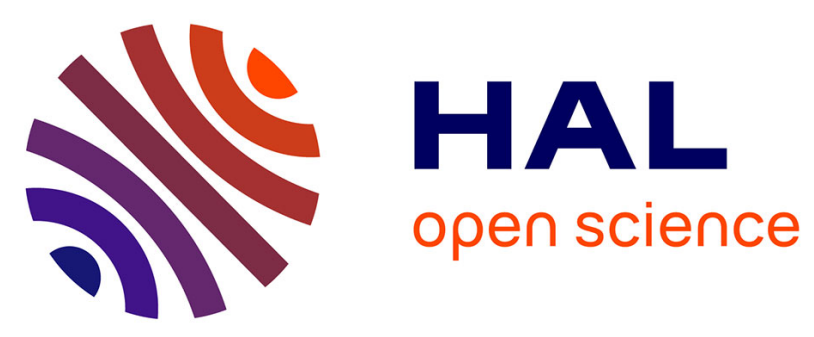

\title{
Imperfect Hele - Shaw cells
}

P.-G. de Gennes

\section{To cite this version:}

P.-G. de Gennes. Imperfect Hele - Shaw cells. Journal de Physique, 1986, 47 (9), pp.1541-1546. 10.1051/jphys:019860047090154100 . jpa-00210353

\section{HAL Id: jpa-00210353 https://hal.science/jpa-00210353}

Submitted on 1 Jan 1986

HAL is a multi-disciplinary open access archive for the deposit and dissemination of scientific research documents, whether they are published or not. The documents may come from teaching and research institutions in France or abroad, or from public or private research centers.
L'archive ouverte pluridisciplinaire HAL, est destinée au dépôt et à la diffusion de documents scientifiques de niveau recherche, publiés ou non, émanant des établissements d'enseignement et de recherche français ou étrangers, des laboratoires publics ou privés. 
Classification

Physics Abstracts

$05.20-47.15-68.45 \mathrm{G}-75.60$

\title{
Imperfect Hele - Shaw cells
}

\author{
P. G. de Gennes \\ Collège de France, 75231 Paris Cedex 05, France
}

(Reçu le 13 février 1986, accepté le 21 mai 1986)

\begin{abstract}
Résumé. - Les surfaces d'une cellule de Hele-Shaw peuvent être dépolies, ou modifiées par des taches de contamination chimique qui modifient les propriétés de mouillage. Nous analysons ici en premier l'injection quasi statique d'un fluide (partiellement mouillant $=$ angle de contact $\theta_{e}$ fini) dans une telle cellule. La forme de la région injectée dans ce milieu bidimensionnel aléatoire dépend beaucoup d'un paramètre $\tau$ (rapport énergie de ligne/énergies de fluctuations dans une tache). Pour $\tau \rightarrow 0$, on doit avoir un amas de percolation et un comportement de matériaux « doux ». Pour $\tau \widetilde{>} 1$, on attend un comportement « dur », avec une pénétration qui ne prend place qu'au-dessus d'une certaine pression coercitive $p_{\mathrm{m}}$, et une frontière presque rectiligne. Dans le cas « doux », nous discutons aussi les écoulements unidimensionnels à vitesse $U$ finie mais faible (ou à nombre capillaire $C a \ll 1$ ). La largeur $l$ du front entre régions sèches et humides doit être proportionnelle à $\mathrm{Ca}^{-1}$. La taille $\xi$ des amas les plus grands croissant depuis l'interface moyenne est plus petite que $l$, et a un exposant d'échelle différent.
\end{abstract}

Abstract. - The covering surfaces of a Hele-Shaw cell may be roughened mechanically, or modified by random chemical patches which change the wetting properties. We discuss first the quasi-static injection of a single fluid (with a finite contact angle $\theta_{\mathrm{e}}$ ) in an imperfect cell. The shape of the injected region depends critically on a dimensionless number $\dot{\tau}$ (ratio of the boundary energy $\tau$ over the capillary energy fluctuations in one patch). At $\tau \ll 1$ we expect a « soft " penetration in the form of percolation clusters. At $\tau \widetilde{>} 1$ we expect a « hard » behaviour; penetration should occur only above a certain coercive pressure, and the boundary is much more stiff. In the soft case, we also consider one dimensional flows, where the fluid advances with a small but finite velocity $U$ (or capillary number $\mathrm{Ca}$ ). The width $l$ of the front between dry and wet regions should scale like $\mathrm{Ca}^{-1}$. The size $\xi$ of the largest clusters sprouting from the interface is smaller than $l$ and scales with a different exponent.

\section{Two types of disorder.}

An incompressible fluid moving in a narrow gap (thickness $h$ ) between two parallel plates, gives rise to simple potential flows [1]. When the fluid is injected in an empty cell, the interface is stable, but when the injected fluid pushes out a second liquid of higher viscosity, a classic instability sets in [2]. It has recently been noticed that modifications of the plate surfaces may then affect the interfacial pattern [3]. In the present note, we analyse some elementary effects of two types of randomness :

(A) Geometrical disorder : the upper plate, for instance, instead of being planar $(z=h)$, is defined by an irregular surface :

$$
z=h+\zeta(x, y) \quad(|\zeta| \ll h) .
$$

(B) Chemical disorder : the interfacial tensions $\gamma_{s 1}\left(\gamma_{s 2}\right)$ between the upper surface $S$ and liquid 1(2) are modulated :

$$
\gamma_{s 1}-\left.\gamma_{s 2}\right|_{x, y}=\bar{\gamma}_{s 1}-\bar{\gamma}_{s 0}+\Delta(x y) .
$$

\section{Static equilibrium.}

Consider the interface between a fluid (1) at uniform pressure $p$ and a fluid (2) at pressure 0 . The equilibrium conditions correspond to $a$. force balance along a horizontal direction $\mathbf{n}\left(n_{x}, n_{y}\right)$ normal to the interface (Fig. 1). For case A this gives :

$$
p(h+\zeta)=p_{0} h+\gamma \frac{\mathrm{d} \zeta}{\mathrm{d} n} \sin \theta_{\mathrm{e}} \quad\left(\frac{\mathrm{d} \zeta}{\mathrm{d} n} \ll 1\right)
$$

Here $\gamma$ is the $1-2$ interfacial tension, and $p_{0}$ is the equilibrium pressure for a smooth cell :

$$
p_{0} h=-2 \gamma \cos \theta_{\mathrm{e}} \equiv 2\left(\gamma_{\mathrm{s} 1}-\gamma_{\mathrm{s} 2}\right)
$$

$\theta_{\mathrm{e}}$ being the contact angle : the second equality expresses the classical Young condition for $\theta_{\mathrm{e}}$. 

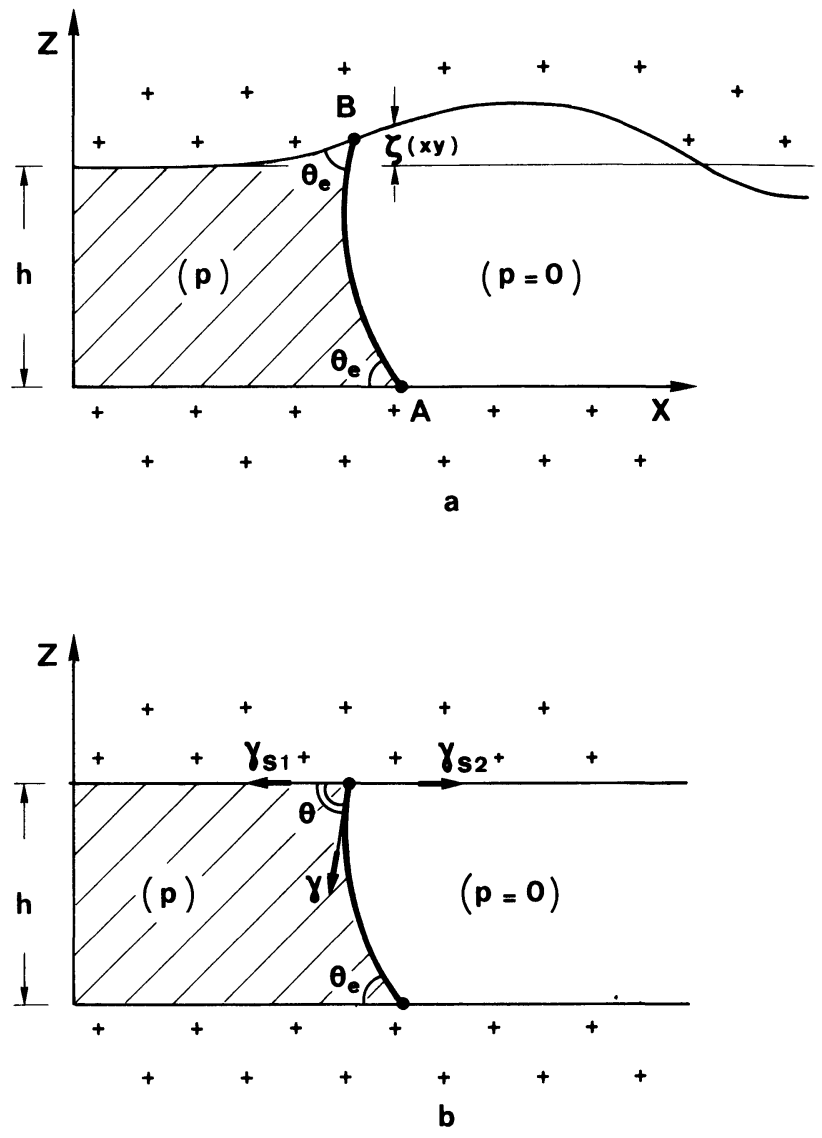

Fig. 1. - Local equilibrium of an interface in Hele-Shaw cell with modulations of the upper surface : a) corrugations; b) chemical alterations changing the interface tensions $\gamma_{s 1}, \gamma_{s 2}$

We now make two simplifying assumptions : (a) $\theta_{\mathrm{e}}$ is not equal to $\pi / 2$, and $p_{0}$ is thus finite $\left(p_{0} \sim \gamma / h\right)$.

(b) The correlation length $d$ of the random variable $\zeta(x, y)$ is much larger than the cell thickness $h$. Then

$$
\gamma \frac{\mathrm{d} \zeta}{\mathrm{d} n} \sim \gamma \zeta / d \ll p \zeta \sim p_{0} \zeta \sim \gamma \zeta / h
$$

and equation (3) is greatly simplified :

$$
h p=h p_{0}(1-\zeta / h)+\frac{J}{\rho} \quad(p \gg h) .
$$

In equation (4) we have added a term $\mathfrak{J} / \rho$ describing the contribution from a curvature of the interfacial " line " (actually a stripe of interface seen as a line in the $x, y$ plane). The curvature $\rho^{-1}$ is counted positive when fluid 1 is surrounded by $2 . J$ is the energy associated with the stripe :

$$
J=\gamma h \frac{\pi-2 \theta_{\mathrm{e}}}{2 \cos \theta_{\mathrm{e}}}
$$

For case B (Fig. 1b), assuming (as an example) that only the upper surface is polluted, we get the force balance

$$
h\left(p-p_{0}\right)=\Delta+\frac{J}{\rho} \quad(\text { case B }) .
$$

We see that both cases are equivalent (when $d \gg h$ ), the correspondence being $-p_{0} \zeta \leftrightarrow \Delta$.

\section{Quasi-static filling by a single fluid.}

We now consider a certain amount of fluid 1 being injected very slowly in the cell, either from a point source, or from a wall. We replace fluid 2 by air - i.e. by a passive, infinitely compressible, non viscous gas : this removes the Saffman Taylor instability, and also the trapping effects found by Lenormand [4] in $2 \mathrm{~d}$ porous systems when fluid (2) is surrounded by fluid (1). Depending on the size $d$ of the patches, two very different behaviours are expected :

3.1 WEAK TENSION. - If the size $d$ of the correlated regions is large enough, the curvature term in equations (4), (6) (which is as most of order $\mathrm{J} / d$ ) becomes negligible. More precisely we can define a reduced line tension

$$
\tau=\frac{J}{d \Delta_{\mathrm{m}}}
$$

where $\Delta_{\mathrm{m}}$ is the r.m.s. average of $\Delta(x, y)$.

If $\tau$ is small, equation (6) (with $J / \rho \rightarrow 0$ ) shows that the wet regions correspond to $\Delta<h\left(p-p_{0}\right)$. The interface is a line of constant $\Delta$ (or constant $\zeta$ ).

This simple geometrical rule has far reaching consequences :

a) link with percolation clusters : the regions of low $\Delta(x, y)$, which may be wet according to the rule, are in the form of « lakes » at low $p$. But when $p$ is above a certain threshold $p_{c}$, we can also have an « ocean ». Consider for instance injection from a point source : at $p<p_{\mathrm{c}}$ we fill one single lake, or percolation cluster; at $p>p_{\mathrm{c}}$ we fill an infinite cluster. On the other hand, if we inject from a wall (say from the plane $x=0$ ) all clusters connected to this wall are filled : below $p_{\mathrm{c}}$ they are restricted to a certain strip, of width $x$ equal to the correlation length. Above $p_{\mathrm{c}}$ the infinite cluster invades all the cell. This situation is similar to the quasi-static entry of a non wetting fluid in a porous material [5-7].

b) Wiggly interface : if we inject from a point source, and impose a pressure just below $p_{\mathrm{c}}$, we generate a finite but large cluster, with a fractal dimensionality [9].

$$
D=2-\beta / v \cong 1.9
$$

where $\beta$ and $v$ are standard, 2 dimensional percolation exponents, defined for instance in reference [9]. $D$ is close to 2 , and thus the interface is very wiggly. 
c) Irreversibility: if we increase the pressure at the source up to a value $p_{\mathrm{m}}$, and then decrease it back to $p=0$, do we recover an empty cell ? To answer is no. When we raise the pressure up to a certain value $p^{\prime}$ our lake may reach the altitude of a surrounding mountain pass, and then spill in a neighbouring valley. When we later decrease the pressure below $p^{\prime}$, the neighbouring valley remains wet.

d) First saturation curve : we are often interested in regimes where the pressure $p$ is monotonously increasing. We can then measure a first saturation curve $\Phi_{+}(p)$, giving the fraction of the cell which is wet, as a function of applied pressure. The general aspect of $\Phi_{+}$ is shown in figure 2. For a macroscopic cell, we have no filling below the percolation threshold $p=p_{\mathrm{c}}$. Above $p_{\mathrm{c}}$ we saturate, in a pressure interval $\delta p$. From equations (4), (6) we have

$$
\delta p \sim \Delta_{\mathrm{m}} / h .
$$

Thus $\frac{\delta p}{p_{0}} \sim \frac{\Delta_{\mathrm{m}}}{\gamma}$ will be smaller than unity. The fraction $\Phi_{+}(p)$ corresponds to the weight of the infinite cluster. Near threshold $\left(p \rightarrow p_{\mathrm{c}}\right)$ it should thus show a power law behaviour [9]

$$
\Phi_{+} \cong\left(\frac{p-p_{\mathrm{c}}}{\delta p}\right)^{\beta} \quad \beta \cong 0.17 .
$$

One important feature of figure 2 is that, as soon as we impose an increase in pressure $\mathrm{d} p$ (above $p_{\mathrm{c}}$ ) we get an increase in saturation $\left(\mathrm{d} \Phi_{+} / \mathrm{d} p>0\right)$. There is no coercive force. In analogy with magnetic materials, we may say that the random structure is soft.

3.2 STRong tensions $(\tau \gg 1)$. - The fractal clusters which we discussed above would have a large line energy, and are thus forbidden when $\tau$ is large. This regime is rather complex and we do not understand it completely, but the following remarks emerge.

a) Differences with the Imry-Ma problem. The equilibrium statistics of a Bloch wall in an Ising system of spins with random fields has been discussed in a classic paper by Imry and Ma [10]. Starting (in 2 dimensions) with a straight boundary of length $L \gg d$ they find that the lowest energy conformation has lateral fluctuations (induced by the randomness) of amplitude

$$
W=L \tau^{-2 / 3} .
$$

(This holds provided that $W \gg d$, or $L \gg d \tau^{2 / 3}$.)

We do not think that the result [9] can be transposed exactly to our problem, where the boundary advances irreversibly. The line is trapped by certain barriers, and need not be in the deepest minimum. We shall try and discuss some of these barriers below;

b) The anchoring length $\lambda^{*}$. Consider the situation depicted in figure 3 , where the line has been anchored
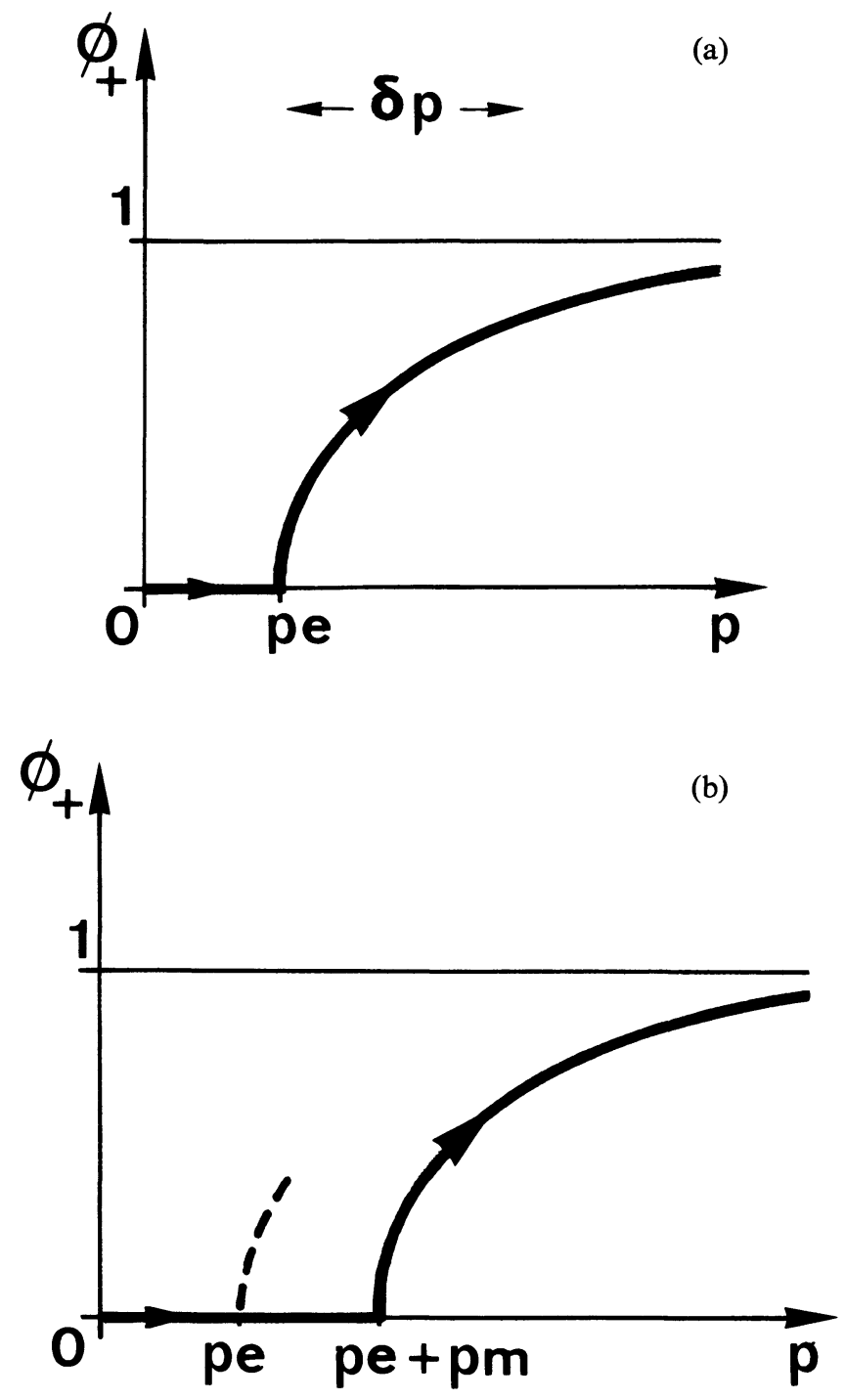

Fig. 2. - First saturation curve : filling fraction $\Phi_{+}$under increasing pressures $p$. a) The " soft" case $(\tau \ll 1)$ with a percolation threshold $p_{c}$ b) The « hard " case $(\tau \gg 1)$ with a coercive pressure $p_{\mathrm{c}}+p_{\mathrm{m}}$. Note that these figures are purely qualitative. In practice the interesting pressure interval $(\delta p)$ will often be much narrower than the equilibrium pressure $\left(p_{0}\right)$ : see the discussion before equation (8).

on a large defective region of linear size $\lambda$. We have a large $\tau$, and we expect that the line is not wiggly at scales below $\lambda$. The total curvature force to the right, coming from the region $\mathrm{AB}$, is of order $\tau$. Along the arc $\mathrm{AB}$, the line faces $N=\lambda / d$ independent patches, each giving a force $\pm \Delta_{\mathrm{m}} d$. The average barrier force along this arc is thus $F_{\mathrm{B}}=N^{1 / 2} \Delta_{\mathrm{m}} d$. We then find two cases

- if $\lambda<\lambda^{*}=\mathrm{d} \tau^{2}$ the tension $\tau$ dominates over the barrier force $F_{\mathrm{B}}$ and the $\mathrm{AB}$ region will move : the line will return to a straight conformation;

- if $\lambda>\lambda^{*}$ the tension cannot overcome the barrier : the line is pinned. Then we expect a boundary which is stiff at small scales. 


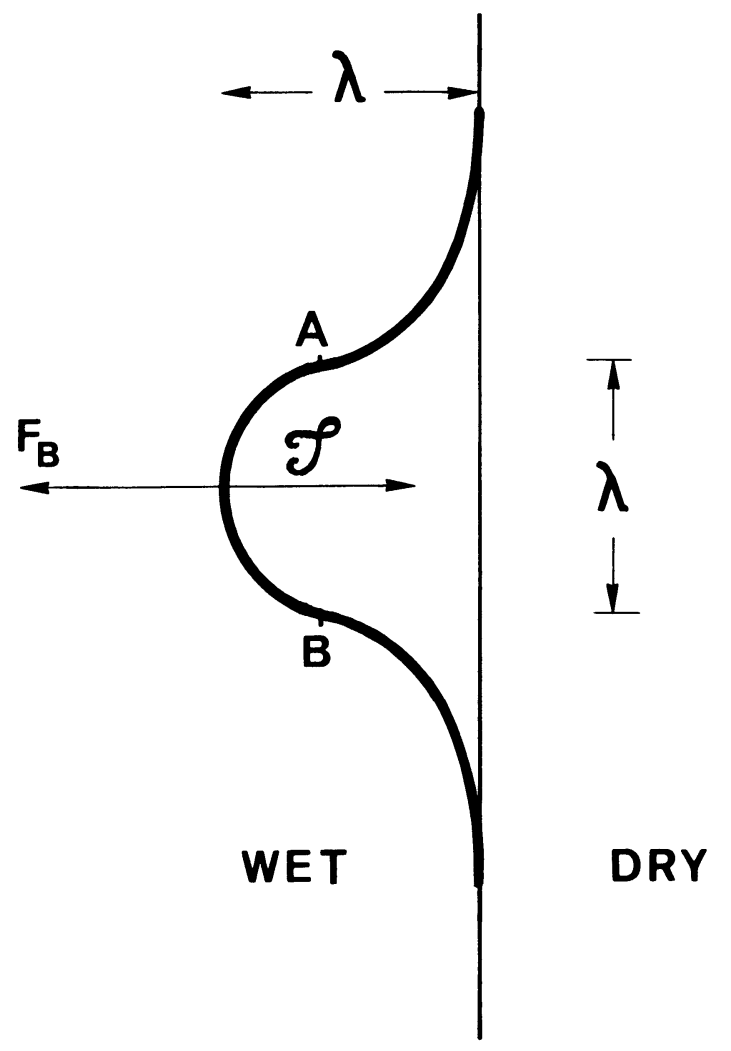

Fig. 3. - Retraction of an anchored region (AB) of linear size $\lambda$. The line tension $\tau$ favours retraction. But the first barriers create a pinning force $F_{\mathrm{B}}$. At large scales $\left(\lambda>\lambda^{*}\right)$ $F_{\mathbf{B}}$ is larger than $\tau$.

Note the difference with the Imry-Ma argument : we do not compare the energies in the anchored state and in the straight state. We look at the local force, i.e. at the first barrier available.

c) The coercive pressure $p_{\mathrm{m}}$. At high $\tau$, with a locally stiff boundary, we expect to have a " hard" material, with a finite coercive field (in magnetic language). The first saturation curve $\Phi_{+}(p)$ starts not at the value $p_{\mathrm{c}}$ defined in the " soft " limit, but at a higher pressure $p_{\mathrm{c}}+p_{\mathrm{m}}$ (Fig. 2b). We estimate the coercive pressure $p_{\mathrm{m}}$ by the following (tentative) argument.

The portions of boundary line to be moved are at least of length $\lambda^{*}$. The corresponding barrier force is $F_{\mathbf{B}} \sim \tau$. Thus the coercive pressure will compensate the barrier force if $p_{\mathrm{m}} h \lambda^{*} \sim \tau(\sim \gamma h)$. This gives

$$
p_{\mathrm{m}} \sim \frac{\gamma}{\mathrm{d} \tau^{2}} \sim \frac{\Delta_{\mathrm{m}}^{2}}{\gamma} \frac{d}{h^{2}}
$$

Thus, for $\tau>1$, at fixed $\gamma$ and $d, p_{\mathrm{m}}$ is expected to be a decreasing function of the reduced line tension $\tau$. At first sight this may appear surprising. However, the trend is plausible. When $\tau$ increases, the basic segment (of length $\lambda^{*}$ ) increases, and the relative fluctuations in the pinning force get smaller and smaller. Ultimately, at very large $\tau$, the line becomes completely straight, and the fluctuations are negligible : the coercive pressure $p_{\mathrm{m}}$ vanishes.

Consider now the complete interval allowed for $\tau$, namely $0<\tau<\infty$. We expect that the coercive pressure $p_{\mathrm{m}}(\tau)$ will increase first from $p_{\mathrm{m}}(0)=0$ to a maximum around $\tau=1\left[p_{\mathrm{m}}(1) \sim \gamma / d\right]$. Then at higher $\tau$, fluctuations are suppressed and $p_{\mathrm{m}}(\tau)$ decreases as suggested by equation (10). In fact, at high $\tau, p_{\mathrm{m}}$ should rapidly become undetectable.

\section{Slow motions of a single fluid.}

We now focus our attention on the case $\tau<1$, where the quasi-static interface is very wiggly, But the liquid (1) is now injected from one extremity of the cell, with an imposed average velocity $U$; again we assume that the liquid pushes a passive gas, of negligible mechanical effects. We consider only very low capillary numbers $C a=U \eta / \gamma$ ( $\eta$ being the viscosity of fluid 1). At $C a \ll 1$, the interface will still be rather irregular, with a large overall width $l$. Our approach to this problem follows the tradition of porous media [11-12], with the following ingredients :

a) use of a coarse-grained profile $\Phi(x, t)$ giving the filling fraction at a distance $x$ from the injecting boundary (this coarse grained description being acceptable if $l \gg d$ )

b) a quasi-static relation between the local filling fraction and the local pressure $p:$ since $p$ is always increasing, we use the curve $\Phi_{+}(p)$ of figure $2 \mathrm{a}$;

c) a generalized Darcy law relating the liquid current $\left(J_{x}, J_{y}\right)$ to the pressure gradient $\nabla p$

$$
\mathbf{J}=-K(\Phi) \nabla p .
$$

In the low $(\mathrm{Ca})$ regime, the permeability $K$ is entirely due to flows along the backbone of the infinite cluster. Furthermore, because we assumed $h \ll d$, the permeability should scale like the electrical conductance of the wet regions. Near threshold, this electrical conductance is described by a certain exponent $\tilde{t}$, specific of continuous percolation problems [13]. However, in two dimensions, the conclusion is that $t$ does not differ significantly from the standard conductivity exponent $t(\cong 1.1$ to 1.3$)$ for discrete percolation problems [9]. Thus the scaling form of $K$ near threshold is expected to be

$$
K(\Phi) \cong \frac{h^{3}}{\eta_{1}}\left(\frac{p-p_{\mathrm{c}}}{\delta p}\right)^{t} \cong \frac{h^{3}}{\eta_{1}} \Phi^{t / \beta}
$$

where $\eta_{1}$ is the viscosity of the fluid.

Combining assumptions $(\mathrm{a}, \mathrm{b}, \mathrm{c})$ we are led to a non linear diffusion equation :

$$
\frac{\partial \Phi}{\partial t}=-\nabla[D(\Phi) \nabla \Phi]
$$


The diffusion coefficient

$$
D(\Phi)=K(\Phi) h^{-1} \frac{\mathrm{d} p}{\mathrm{~d} \Phi_{+(p)}}
$$

has the limiting behaviour :

$$
\begin{aligned}
D(\Phi) & \cong \frac{\Delta_{\mathrm{m}} h}{\eta} \Phi^{\mu} \quad(\Phi \rightarrow 0) \\
\mu & =\frac{t+1}{\beta}-1 .
\end{aligned}
$$

If $t \sim 1.3$, the exponent $\mu$ is very large $(\sim 15)$.

Let us now consider specifically a steady state interface, moving at constant velocity $U$

$$
\frac{\partial \Phi}{\partial t} \rightarrow-U \frac{\partial \Phi}{\partial x}
$$

The resulting profile $\Phi(x)$ is qualitatively shown in figure 4 , and ruled by

$$
-\frac{D(\Phi) \mathrm{d} \Phi}{U \Phi}=\mathrm{d} x
$$

The overall width of the profile is

$$
l \cong \frac{\bar{D}}{U} \quad \bar{D}=\frac{\Delta_{\mathrm{m}} h}{\eta} .
$$

In equation (16) we made use of our earlier estimate of the pressure interval (described before Eq. (8)).

The profile has a sharp edge at $x=x_{\mathrm{m}}(\Phi=0)$ because $D(\Phi=0)=0$. This corresponds to a « hypodiffusive " case in the notation of reference [12]. Near $x=x_{\mathrm{m}}$ the profile derived from (13) and (16) is

$$
\Phi \cong\left[\frac{U\left(x_{\mathrm{m}}-x\right)}{\bar{D}}\right]^{1 / \mu}
$$

All the above discussion was restricted to the coarse grained profile $\Phi(x, t)$. Of course, the microscopic interface will show fluctuations, resembling percolation clusters sprouting from an average front. We can get the characteristic size $\xi$ of these clusters through the conditions $[8,14]$

$$
\left\{\begin{aligned}
\xi & =\mathrm{d} \Phi^{-v / \beta} \\
\Phi & =\Phi\left(x=x_{\mathrm{m}}-\xi\right)
\end{aligned}\right.
$$

where $\Phi$ is computed from the average profile (17), while $v \sim 4 / 3$ is the correlation length exponent for $2 d$ percolation.

The result is :

$$
\begin{aligned}
& \frac{\xi}{l} \cong\left(\frac{d}{l}\right) p \\
& \frac{1}{p}=1+\frac{v}{\beta \mu}
\end{aligned}
$$
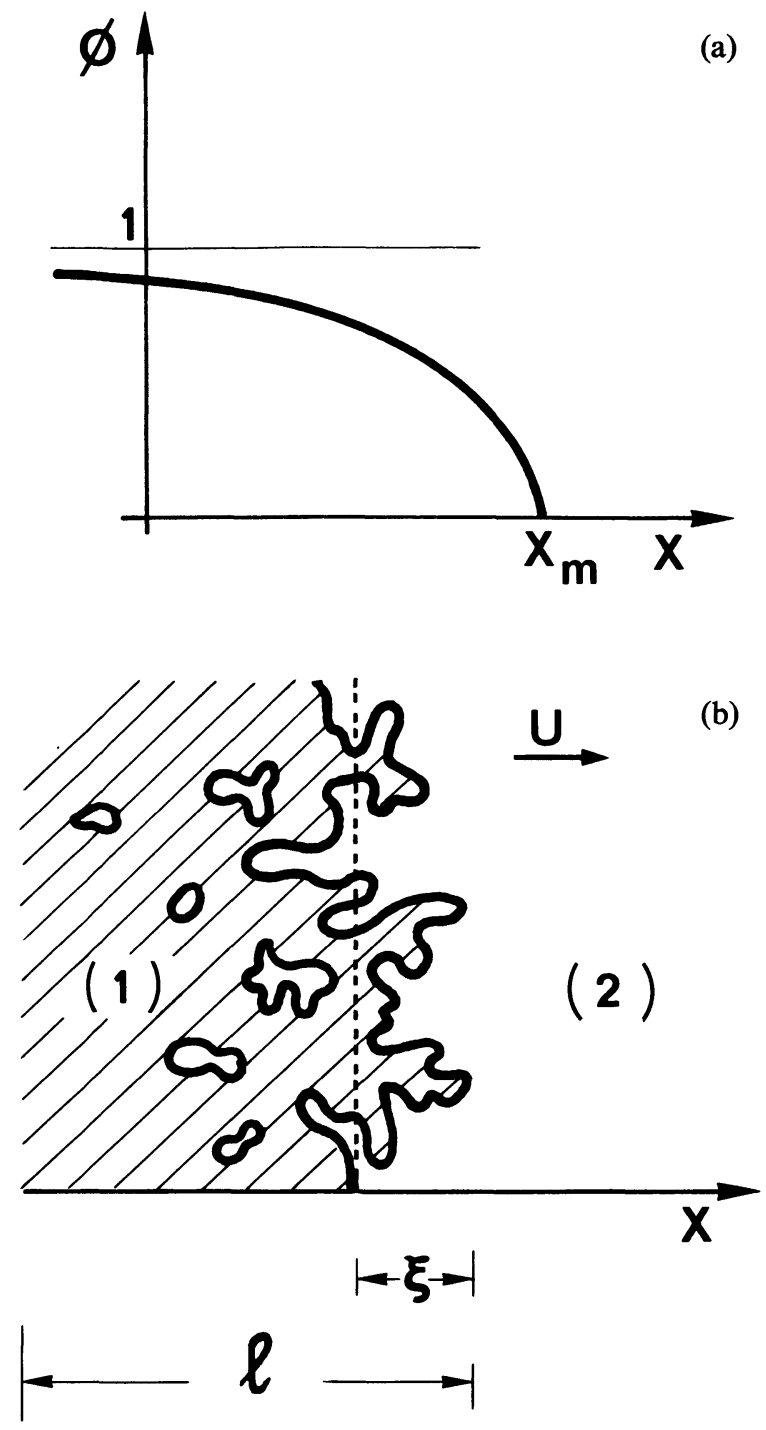

Fig. 4. - Steady state injection of a fluid in an empty cell, at low velocities $U$. a) The coarse grained profile, with a width $l$ and a well defined edge $x_{\mathrm{m}}$ (hypodiffusive regime). b) A qualitative sketch of the actual situation showing the region of strong fluctuations, with a width $\xi$, defined by equation (16).

In the limit of low capillary numbers $\xi / l$ is thus small : the coarse grained description is sufficient, except in the near vicinity of the edge $\left(x \rightarrow x_{\mathrm{m}}\right)$. Note again that all our discussion of the dynamics $(v \neq 0)$ is restricted to the soft regime $(\tau \ll 1)$. In the opposite case $(\tau \gg 0)$ the dynamics are not at all understood.

\section{Conclusions.}

1) Imperfect Hele-Shaw cells may become a useful model for porous media - for two reasons :

a) the randomness is described by one function $\Delta(x y)$ or $\zeta(x y)$

b) the permeability problem is decoupled from the energy barrier problem (the hydrodynamic thickness of the channels is always $h$ ). 
2) We have a relatively clear scheme to predict the effects or surface irregularities in a Hele-Shaw cell, when we inject a single liquid, and when we have relatively large patches (large $d$ ) or low reduced tensions $(\tau<1)$. In a quasi-static regime we expect that the fluid spot is irregular like a $2 d$ percolation cluster. In a steady state flow with low capillary numbers, we expect a diffuse front, of width $l$ given by equation (16), and a remnant of the percolation clusters showing up with the scale $\xi$ (Eq. (19)).

On the other hand, there are certain difficulties : in static experiments, all the action takes place within a pressure interval $\delta p \sim \Delta_{\mathrm{m}} / h$ which may be rather small if $h$ corresponds to a usual Hele-Shaw cell $(h \sim 1 \mathrm{~mm})$. The proposed experiments will require thinner cells : $h \sim 50 \mu$ being a plausible value. The pressures are then enhanced, but of course the mechanical rigidity of the whole structure requires some care. The whole cell is now more similar to what is used in studies on nematic liquids (with horizontal dimensions $L \sim 1 \mathrm{~cm}$ ) than to a conventional HeleShaw apparatus $(L=50 \mathrm{~cm})$.

3) What are the modifications expected when we deal with two incompressible fluids ? A minimum of three new effects should come into play :

a) Trapping : when a cluster of (1) surrounds completely a region of (2), this region is trapped (at low capillary numbers, when the cluster structure does not drift in the flow). In $2 d$ this effect is essential, as first pointed out by Lenormand [4]. It has been analysed by Wilkinson [15]. For the 2 dimensional case the percolation thresholds for both liquid(1) and liquid (2) merge, and the cluster of (1) building up in static injection from a source belongs to a new universality class. b) Shock waves : at finite $U$, if we write the adequate (two-fluid) form of the (1 dimensional) coarse grained transport equations, we often find wavelets propagating with a celerity $c(\Phi)$ which is maximal at some intermediate $\Phi$ value : this leads to a shock structure, discussed long ago by Buckley and Leverett [6]. The shock is broadened by diffusion; the order of magnitude of the resulting width is still given by equation (16).

c) Saffman-Taylor instabilities [2] (when the viscosity ratio $\eta_{2} / \eta_{1}$ is larger than unity) : in an ideal HeleShaw cell, this fingering instability extends down to a minimum wavelength $\lambda_{\mathrm{m}} \sim h \mathrm{Ca}^{-1 / 2}$.

In an imperfect cell, at low $C a$, we expect that the essential feature will be the diffusional width $l$ of equation (16). Modes of wave length smaller than $l$ are probably damped out by diffusion, and it is natural to assume that

$$
\lambda_{\mathrm{m}} \cong l \cong h \frac{\Delta_{\mathrm{m}}}{\gamma}(C a)^{-1}
$$

3) On the whole, the main conclusion is that we should determine first all the features of the one liquid problem, and proceed later towards the two liquid problem, separating carefully the three complications listed above : this program being rather different from what is currently done.

\section{Acknowledgments.}

I have benefited from very stimulating discussions with D. Andelman, J. Gollub, H. Hermann, H. Nakanishi, M. Robbins and D. Weitz.
[1] Hele-Shaw, J. S., Nature 58 (1898) 34.

[2] Saffman, P. G., Taylor, G. I., Proc. R. Soc. A 245 (1958) 312.

Park, G. W., Homsy, G. M., J. Fluid Mech. 139 (1984) $291 ; 141$ (1984) 257.

[3] Ben Jacob, E. et al., Phys. Rev. Lett. 55 (1985) 1315.

[4] Lenormand, R., Bories, S., C.R. Heb. Séan. Acad. Sci. 2913 (1980) 279.

LenORMAND, R., ZARCONe, C., SARR, A., J. Fluid Mech. 135 (1983) 337.

Lenormand, R., Zarcone, C., Phys. Chem. Hydrodynamics, to be published (1985).

[5] Guyon, E., DE Gennes, P. G., J. Mécanique 17 (1978) 403.

Guyon, E., Hulin, J. P., Lenormand, R., Ann. Mines (1984) 17.

Dullien, F., Porous media (Acad. Press, N 1) 1979.
[6] Chatzis, I., Dullien, F., Rev. Instrum. Franç. Petr. 37 (2) (1982) 183.

[7] Wilkinson, D., Willemsen, J. F., J. Phys. A 16 (1983) 3365 .

[8] Wilkinson, D., Phys. Rev. A 30 (1984) 520.

[9] StaufFer, D., Introduction to Percolation theory (Taylor and Francis) 1985.

[10] Imry, J., MA, S. K., Phys. Rev. Lett. 35 (1975) 1399.

[11] De Gennes, P. G., Physicochem. Hydrodyn. 4 (1983) 175.

[12] De Gennes, P. G., in Physics of disordered materials, Adler, Fritzsche, Ovshinsky eds (Plenum) 1985, p. 227.

[13] Halperin, B., Feng, S., Sen, P., Phys. Rev. Lett. 54 (1985) 2391.

[14] De GenNes, P. G., Oral presentation at the Schlumberger colloquium on porous media, Ridgefield 1983. 